\title{
INVERSE KINEMATIC AND DYNAMIC ANALYSIS OF REDUNDANT MEASURING MANIPULATOR BKHN-MCX-04
}

\author{
Nguyen Van Khang ${ }^{1}$, Nguyen Phong Dien ${ }^{1}$, \\ Nguyen Van Vinh ${ }^{1}$, Tran Hoang Nam ${ }^{2}$ \\ ${ }^{1}$ Hanoi University of Technology \\ ${ }^{2}$ Vinh Long Pedagogical and Technical College
}

\begin{abstract}
This paper deals with the problem of inverse kinematics and dynamics of a measuring manipulator with kinematic redundancy which was designed and manufactured at Hanoi University of Technology for measuring the geometric tolerance of surfaces of machining components. A comparison between the calculation result and the experimental measurement is also presented.
\end{abstract}

\section{INTRODUCTION}

Robotic systems are coming into general use in the manufacturing industry for measuring geometric tolerances of manufactured products. These robots are equipped with a measuring system and can be used very flexibly for complicated measuring tasks, in particular at locations that are difficult to access.

In the past few years the robotics community evolved growing interest in measuring manipulators which have the characteristic of kinematic redundancy to offer greater flexibility. A kinematically redundant manipulator is a serial robotic arm that has more independently driven joints than necessary to define the desired pose (position and orientation) of its end-effector. In other words, a manipulator is said to be redundant when the dimension of the workspace $m$ is less than the dimension of the joint space $n$. The extra degree-of-freedom presented in redundant manipulators can be used to avoid obstacles, to increase the workspace or to optimize the motion of the manipulator according to a cost function. Particular attention has been devoted to the study of redundant manipulators in the last twenty years [1-2]. A number of scientific works are focused upon kinematic analysis $[1,3,5,14]$, motion planning $[4,6]$ and controls $[2,7,10]$ of redundant robot manipulators. Summaries of much of the past work are given in refs. [8-12]. Although different methods and solutions have been proposed and reported, the theory related to the problem continues to develop and new approaches are regularly being published.

This paper presents some results of the inverse dynamic analysis and control algorithm of a redundant manipulator called BKHN-MCX-04, which has been designed and manufactured at Hanoi University of Technology for measuring the geometric tolerance of surfaces of machining components. The mechanical model of the measuring manipulator is 
introduced in Section 2. The inverse kinematic problem of the manipulator is investigated in Section 3. Section 4 presents the results of the inverse dynamic analysis. Finally, the experimental investigation to verify the obtained theoretical results is given in Section 5 .

\section{MECHANICAL MODEL OF THE MEASURING MANIPULATOR}

Fig. 1 shows the mechanical model of the manipulator BKHN-MCX-04 as an open kinematic chain of rigid bodies. The manipulator is driven directly by six servomotors. The first motor drives link 1 rotating about the vertical axis $z_{0}$. Rotating axes of the next three motors which drive links 2, 3 and 4 are parallel. The fifth servomotor drives link 5 to rotate about the link axis. Links 2, 3, 4 and 5 are assumed to move in a plane. While the first four motors are used to manipulate point $\mathrm{O}_{5}$ moving along a prescribed trajectory corresponding to the measuring task, the fifth motor changes the orientation of link 5 to accord with the measuring surface. The last motor located at $\mathrm{O}_{5}$ drives the endeffector link 6 to come into contact with the measuring surface. With such configuration, the manipulator is able to perform flexibly measurements for geometrically complicated surfaces. Design parameters of the manipulator are given in Tab. 1.

Table 1. Design parameters of the manipulator

\begin{tabular}{|c|c|}
\hline link $i$ & Distance $\mathrm{O}_{i-1} \mathrm{O}_{i}(\mathrm{~m})$ \\
\hline 1 & 0.14 \\
\hline 2 & 0.15 \\
\hline 3 & 0.20 \\
\hline 4 & 0.0 \\
\hline 5 & 0.163 \\
\hline 6 & 0.080 \\
\hline
\end{tabular}

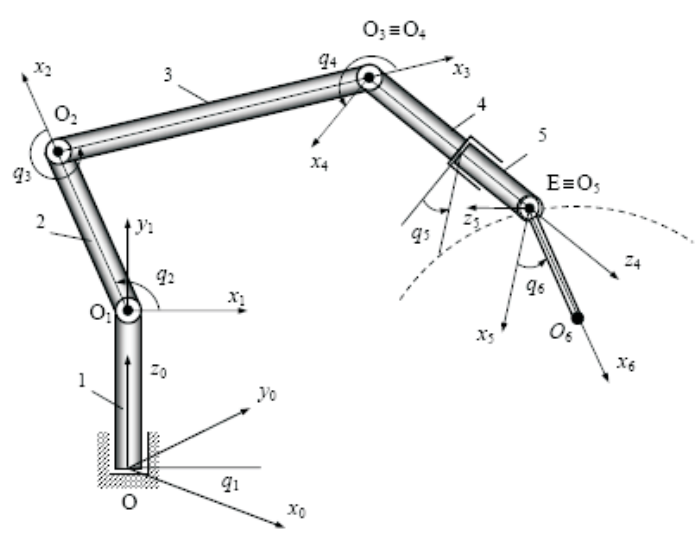

Fig. 1. Structural diagram and coordinate frames of manipulator BKHN-MCX-04

First, we introduce the fixed coordinate frame $\left\{x_{0}, y_{0}, z_{0}\right\}$ located at point $\mathrm{O}$. In addition, the $z_{0}$-axis is chosen to be in line with the first motor axis. For convenience, the 
moving coordinate frame $\left\{x_{i}, y_{i}, z_{i}\right\}$ attached to link $i$ is chosen according to the DenavitHartenberg (DH) notation [9] as shown in the figure. The moving configuration of the manipulator is described by six rotation angles $q_{i}$ (for $i=1,2, \ldots, 6$ ). The position and orientation of the end-effector link 6 can be determined by a set of three coordinates of point $\mathrm{O}_{5}$ and two rotation angle as $q_{5}, q_{6}$. Thus, the dimension of the joint space $n=6$ and the dimension of the workspace $m=5$. The degree of redundancy of the overall system is $r=n-m=1$.

\section{INVERSE KINEMATIC ANALYSIS}

As mentioned in the previous section, the real motion trajectory of point $\mathrm{O}_{5}$ influences essentially the accuracy of measurements. Therefore, the following inverse kinematic analysis deals with the calculation of angles $q_{i}$ (for $i=1,2, \ldots, 5$ ) for a desired motion of point $\mathrm{O}_{5}$. Let $\mathbf{x}=\left[x_{E}, y_{E}, z_{E}\right]^{T}$ be the vector of workspace coordinates (Cartesian variables) of point $E \equiv O_{5}$ in the fixed coordinate frame $\left\{O x_{0} y_{0} z_{0}\right\}$ and $\mathbf{q}=$ $\left[q_{1}, q_{2}, q_{3}, q_{4}, q_{5}\right]^{T}$ the vector of joint angles (joint variables), the degree of redundancy for this case is two. In general, the joint angles $\mathbf{q}$ and workspace coordinates $\mathbf{x}$ are related by the following expression

$$
\mathbf{x}=\mathbf{f}(\mathbf{q})
$$

where $\mathbf{f}$ is a vector function representing the manipulator forward kinematics. Eq. (1) can be derived conveniently using DH-parameters given in Tab. 2, where we use the DHnotation $d_{1}=O O_{1}, a_{2}=O_{1} O_{2}, a_{3}=O_{2} O_{3}, d_{5}=O_{4} O_{5}$.

Table 2. DH-parameters

\begin{tabular}{|l|l|l|l|l|}
\hline Link $i$ & $\theta_{i}$ & $d_{i}$ & $a_{i}$ & $\alpha_{i}$ \\
\hline 1 & $q_{1}$ & $d_{1}$ & 0 & $\pi / 2$ \\
\hline 2 & $q_{2}$ & 0 & $a_{2}$ & 0 \\
\hline 3 & $q_{3}$ & 0 & $a_{3}$ & 0 \\
\hline 4 & $q_{4}$ & 0 & 0 & $-\pi / 2$ \\
\hline 5 & $q_{5}$ & $d_{5}$ & 0 & $\pi / 2$ \\
\hline
\end{tabular}

Thus, let $\mathbf{H}_{i}$ be the $4 \times 4$ matrix transforming the coordinates of a point in the coordinate frame $\left\{x_{i-1}, y_{i-1}, z_{i-1}\right\}$ into the coordinate frame $\left\{x_{i}, y_{i}, z_{i}\right\}$, one obtains

$$
\begin{aligned}
\mathbf{H}_{1} & =\left[\begin{array}{cccc}
C_{1} & 0 & S_{1} & 0 \\
S_{1} & 0 & -C_{1} & 0 \\
0 & 1 & 0 & d_{1} \\
0 & 0 & 0 & 1
\end{array}\right], \mathbf{H}_{2}=\left[\begin{array}{cccc}
C_{2} & -S_{2} & 0 & a_{2} C_{2} \\
S_{2} & C_{2} & 0 & a_{2} S_{2} \\
0 & 0 & 1 & 0 \\
0 & 0 & 0 & 1
\end{array}\right], \mathbf{H}_{3}=\left[\begin{array}{cccc}
C_{3} & -S_{3} & 0 & a_{3} C_{3} \\
S_{3} & C_{3} & 0 & a_{3} S_{3} \\
0 & 0 & 1 & 0 \\
0 & 0 & 0 & 1
\end{array}\right] \\
\mathbf{H}_{4} & =\left[\begin{array}{cccc}
C_{4} & 0 & -S_{4} & 0 \\
S_{4} & 0 & C_{4} & 0 \\
0 & -1 & 0 & 0 \\
0 & 0 & 0 & 1
\end{array}\right], \mathbf{H}_{5}=\left[\begin{array}{cccc}
C_{5} & 0 & S_{5} & 0 \\
S_{5} & 0 & -C_{5} & 0 \\
0 & 1 & 0 & d_{5} \\
0 & 0 & 0 & 1
\end{array}\right] .
\end{aligned}
$$


where we use short notations $S_{i}=\sin \left(q_{i}\right), C_{i}=\cos \left(q_{i}\right)$. The coordinate transformation matrix between the coordinate frame $\left\{O x_{0} y_{0} z_{0}\right\}$ and $\left\{O_{5} x_{5} y_{5} z_{5}\right\}$ takes the form

$\mathbf{D}_{5}=\mathbf{H}_{1} \mathbf{H}_{2} \mathbf{H}_{3} \mathbf{H}_{4} \mathbf{H}_{5}$

$$
=\left[\begin{array}{cccc}
C_{1} C_{234} C_{5}-S_{1} S_{5} & -C_{1} S_{234} & S_{1} C_{5}+C_{1} S_{5} C_{234} & -d_{5} C_{1} S_{234}+a_{2} C_{1} C_{2}+a_{3} C_{1} C_{23} \\
S_{1} C_{234} C_{5}+C_{1} S_{5} & -S_{1} S_{234} & -C_{1} C_{5}+S_{1} S_{5} C_{234} & -d_{5} S_{1} S_{234}+a_{2} S_{1} C_{2}+a_{3} S_{1} C_{23} \\
S_{234} C_{5} & C_{234} & S_{5} S_{234} & d_{1}+d_{5} C_{234}+a_{2} S_{2}+a_{3} S_{23} \\
0 & 0 & 0 & 1
\end{array}\right]
$$

where $S_{i j k}=\sin \left(q_{i}+q_{j}+q_{k}\right)$ and $C_{i j k}=\cos \left(q_{i}+q_{j}+q_{k}\right)$. The use of elements of matrix $\mathbf{D}_{5}$ yields the relationship between joint variables $\mathbf{q}$ and Cartesian variables $\mathbf{x}$

$$
\begin{aligned}
& x_{E}=a_{2} C_{1} C_{2}+a_{3} C_{1} C_{23}-d_{5} C_{1} S_{234} \\
& y_{E}=a_{2} S_{1} C_{2}+a_{3} S_{1} C_{23}-d_{5} S_{1} S_{234} \\
& z_{E}=a_{2} S_{2}+a_{3} S_{23}+d_{5} C_{234}+d_{1}
\end{aligned}
$$

Eq. (2) can be expressed in the same matrix form as Eq. (1). Differentiating Eq. (1) with respect to time, we obtain the relation between generalized velocities

$$
\dot{\mathbf{x}}=\mathbf{J}(\mathbf{q}) \dot{\mathbf{q}}
$$

where $\mathbf{J}(\mathbf{q})=\frac{\partial \mathbf{f}}{\partial \mathbf{q}}$ is the Jacobian matrix

$$
J=\left[\begin{array}{lllll}
\frac{\partial x_{E}}{\partial q_{1}} & \frac{\partial x_{E}}{\partial q_{2}} & \frac{\partial x_{E}}{\partial q_{3}} & \frac{\partial x_{E}}{\partial q_{4}} & \frac{\partial x_{E}}{\partial q_{5}} \\
\frac{\partial y_{E}}{\partial q_{1}} & \frac{\partial y_{E}}{\partial q_{2}} & \frac{\partial y_{E}}{\partial q_{3}} & \frac{\partial y_{E}}{\partial q_{4}} & \frac{\partial y_{E}}{\partial q_{5}} \\
\frac{\partial z_{E}}{\partial q_{1}} & \frac{\partial z_{E}}{\partial q_{2}} & \frac{\partial z_{E}}{\partial q_{3}} & \frac{\partial z_{E}}{\partial q_{4}} & \frac{\partial z_{E}}{\partial q_{5}}
\end{array}\right]=\left[\begin{array}{lllll}
J_{11} & J_{12} & J_{13} & J_{14} & J_{15} \\
J_{21} & J_{22} & J_{23} & J_{24} & J_{25} \\
J_{31} & J_{32} & J_{33} & J_{34} & J_{35}
\end{array}\right]
$$

The elements of $\mathbf{J}(\mathbf{q})$ are

$$
\begin{aligned}
& J_{11}=-d_{5} S_{1} S_{234}-a_{3} S_{1} C_{23}-a_{2} S_{1} C_{2} ; \quad J_{12}=-d_{5} C_{1} C_{234}-a_{3} C_{1} S_{23}-a_{2} C_{1} S_{2} ; \\
& J_{13}=-d_{5} C_{1} C_{234}-a_{3} C_{1} S_{23} ; J_{14}=-d_{5} C_{1} C_{234} ; J_{15}=0 \\
& J_{21}=-d_{5} C_{1} S_{234}+a_{3} C_{1} C_{23}+a_{2} C_{1} C_{2} ; J_{22}=-d_{5} S_{1} C_{234}+a_{3} S_{1} S_{23}+a_{2} S_{1} S_{2} ; \\
& J_{23}=-d_{5} S_{1} C_{234}+a_{3} S_{1} S_{23} ; J_{24}=-d_{5} S_{1} C_{234} ; J_{25}=0 ; J_{31}=0 ; \\
& J_{32}=-d_{5} S_{234}+a_{3} C_{23}+a_{2} C_{2} ; J_{33}=-d_{5} S_{234}+a_{3} C_{23} ; J_{34}=-d_{5} S_{234} ; J_{35}=0
\end{aligned}
$$

Due to kinematic redundancy of the manipulator, the general solution of Eq. (3) can be given as follows [4], [7]

$$
\dot{\mathbf{q}}=\mathbf{J}^{+}(\mathbf{q}) \dot{\mathbf{x}}
$$

where $\mathbf{J}^{+}(\mathbf{q}) \in \mathrm{R}^{5 \times 3}$ is the Moore-Penrose pseudo-inverse of matrix $\mathbf{J}(\mathbf{q})$ defined by

$$
\mathbf{J}^{+}(\mathbf{q})=\mathbf{J}^{T}(\mathbf{q})\left[\mathbf{J}(\mathbf{q}) \mathbf{J}^{T}(\mathbf{q})\right]^{-1}
$$

Note that Eq. (4) leads to the least-squares solution that minimizes $\|\dot{\mathbf{x}}-\mathbf{J} \dot{\mathbf{q}}\|$ and gives the minimum joint velocities for the desired workspace velocity [4]. Differentiating Eq. 
(3) again with respect to time, we obtain the relationship between generalized accelerations as

Eq. (6) yields

$$
\ddot{\mathbf{x}}=\mathbf{J}(\mathbf{q}) \ddot{\mathbf{q}}+\dot{\mathbf{J}}(\mathbf{q}) \dot{\mathbf{q}}
$$

$$
\ddot{\mathbf{q}}=\mathbf{J}^{+}(\mathbf{q})[\ddot{\mathbf{x}}-\dot{\mathbf{J}}(\mathbf{q}) \dot{\mathbf{q}}]
$$

The joint angles can be numerically calculated using the difference approximation

$$
\begin{aligned}
\dot{\mathbf{q}}_{k} & =\frac{\mathbf{q}_{k+1}-\mathbf{q}_{k}}{\Delta t}, \\
\dot{\mathbf{x}}_{k} & =\frac{\mathbf{x}_{k+1}-\mathbf{x}_{k}}{\Delta t},
\end{aligned}
$$

Substituting Eqs. (8) and (9) into Eq. (4), one obtains

$$
\mathbf{q}_{k+1}=\mathbf{q}_{k}+\mathbf{J}^{+}\left(\mathbf{q}_{k}\right)\left(\mathbf{x}_{k+1}-\mathbf{x}_{k}\right)
$$

For a given $\mathbf{x}, \dot{\mathbf{x}}, \ddot{\mathbf{x}}$ we can calculate approximately joint variables $\mathbf{q}$ using Eq. (10) and then angular velocities $\dot{\mathbf{q}}$ using Eq. (4), angular acceleration $\ddot{\mathbf{q}}$ using Eq. (7). However, only a rough solution of $\mathbf{q}$ can be found using Eq. (10). A numerical algorithm to improve the exactness of the solution is proposed in [15]. This numerical algorithm is based on the correction of the increment $\Delta \mathbf{q}=\mathbf{q}_{k+1}-\mathbf{q}_{k}$. The numerical results of the inverse kinematic analysis will be presented in section 5 .

\section{INVERSE DYNAMIC ANALYSIS}

The first step of the inverse dynamic analysis is to formulate the differential equations of motion of the manipulator which is generally expressed in the compact matrix form $[8,9,13]$

$$
\mathbf{M}(\mathbf{q}) \ddot{\mathbf{q}}+\mathbf{C}(\mathbf{q}, \dot{\mathbf{q}}) \dot{\mathbf{q}}+\mathbf{g}(\mathbf{q})=\boldsymbol{\tau}
$$

where $\mathbf{M}(\mathbf{q}) \in \mathbf{R}^{6 \times 6}$ denotes an inertia matrix, $\mathbf{C}(\mathbf{q}, \dot{\mathbf{q}}) \dot{\mathbf{q}} \in \mathbf{R}^{6}$ is a torque vector caused by centrifugal and Coriolis forces, $\mathbf{g}(\mathbf{q}) \in \mathbf{R}^{6}$ is a torque gravity vector, and $\boldsymbol{\tau} \in \mathbf{R}^{6}$ represents a joint torque vector. Note that vectors $\mathbf{q}, \dot{\mathbf{q}}, \ddot{\mathbf{q}}$ have been determined from results of the inverse kinematics and link 6 muss be considered in the dynamic calculation. The second step aims at calculating joint torques $\boldsymbol{\tau}$ from the obtained equations of motion corresponding to a desired trajectory of the end-effector.

The following notations are used to derive equations of motion of the manipulator: $m_{i}$ mass of the link $i$

$\mathbf{r}_{i}=\left[x_{C i}, y_{C i}, z_{C i}\right]^{T}$ position vector of the center of mass $C_{i}$ in $\left\{O x_{0} y_{0} z_{0}\right\}$

$\mathbf{r}_{i}^{(i)}=\left[x_{C i}^{(i)}, y_{C i}^{(i)}, z_{C i}^{(i)}\right]^{T}$ position vector of the center of mass $C_{i}$ in $\left\{O x_{i} y_{i} z_{i}\right\}$

$\mathbf{v}_{i}=\dot{\mathbf{r}}_{i}$ velocity of the center of mass $C_{i}$

$\boldsymbol{\omega}_{i}^{(i)}$ angular velocity of link $i$ with respect to $\left\{O x_{i} y_{i} z_{i}\right\}$

$\mathbf{J}_{T i}(\mathbf{q})=\frac{\partial \mathbf{r}_{i}}{\partial \mathbf{q}}$ translation Jacobian matrix of link $i$

$\mathbf{J}_{R i}^{(i)}(\mathbf{q})=\frac{\partial \boldsymbol{\omega}_{i}^{(i)}}{\partial \dot{\mathbf{q}}}$ rotation Jacobian matrix of link $i$

$\mathbf{A}_{i}$ rotation matrix of link $i$ 
$\mathbf{I}_{i}^{(i)}$ inertia matrix of link $i$ with respect to $C_{i}$ in $\left\{O x_{i} y_{i} z_{i}\right\}$

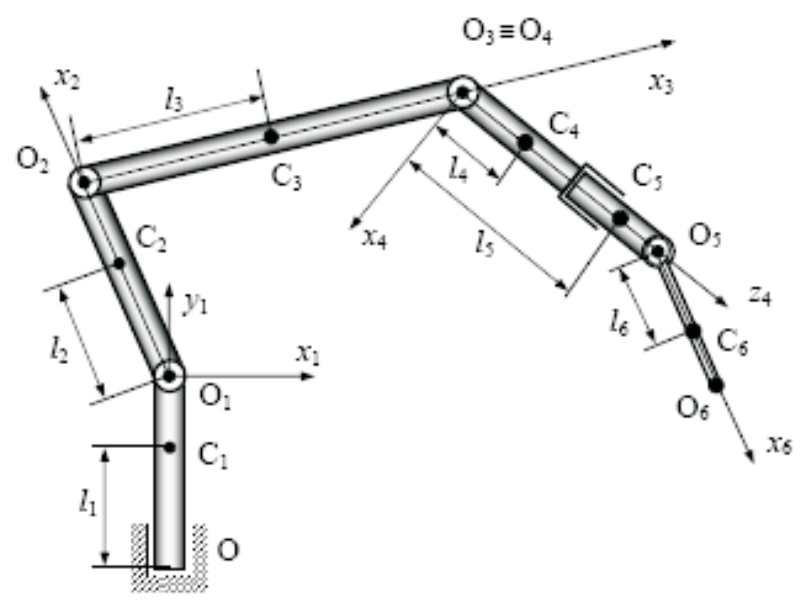

Fig. 2. Position of the centers of mass

The $6 \times 6$ inertia matrix $\mathbf{M}(\mathbf{q})$ is defined by

$$
\mathbf{M}(\mathbf{q})=\sum_{i=1}^{6}\left[\mathbf{J}_{T i}^{T} m_{i} \mathbf{J}_{T i}+\mathbf{J}_{R i}^{(i) T} \mathbf{I}_{i}^{(i)} \mathbf{J}_{R i}^{(i)}\right]
$$
$[13]$

The elements of matrix $\mathbf{C}(\mathbf{q}, \dot{\mathbf{q}})$ can be calculated from $\mathbf{M}(\mathbf{q})$ using the relationship

$$
c_{i j}=\frac{1}{2} \sum_{k=1}^{6}\left(\frac{\partial m_{i j}}{\partial q_{k}}+\frac{\partial m_{i k}}{\partial q_{j}}-\frac{\partial m_{j k}}{\partial q_{i}}\right) \dot{q}_{k}
$$

where $i=1,2, \ldots, 6$ and $j=1,2, \ldots, 6$. The gravity torque vector $\mathbf{g}(\mathbf{q})$ is given by

$$
\mathbf{g}(\mathbf{q})=\frac{\partial \Pi}{\partial \mathbf{q}}
$$

where $\Pi$ denotes the potential energy of the manipulator system.

Firstly, according to Fig. 2, the position vector $\mathbf{r}_{i}$ of the center of mass $C_{i}$ is given by

$$
\mathbf{r}_{i}=\mathbf{r}_{O i}+\mathbf{A}_{i} \mathbf{r}_{i}^{(i)}, \quad(i=1,2, \ldots, 6)
$$

where vectors $\mathbf{r}_{i}^{(i)}$ are design parameters given in Tab. 3 . 
Table 3. Coordinates of the centers of mass

\begin{tabular}{|l|l|l|l|}
\hline \multirow{2}{*}{ Link $i$} & \multicolumn{4}{|l|}{$\mathbf{r}_{i}^{(i)}$} \\
\cline { 2 - 4 } & $x_{C i}^{(i)}$ & $y_{C i}^{(i)}$ & $z_{C i}^{(i)}$ \\
\hline 1 & 0 & $-\left(d_{1}-l_{1}\right)$ & 0 \\
\hline 2 & $-\left(a_{2}-l_{2}\right)$ & 0 & 0 \\
\hline 3 & $-\left(a_{3}-l_{3}\right)$ & 0 & 0 \\
\hline 4 & 0 & 0 & $l_{4}$ \\
\hline 5 & 0 & $-\left(d_{5}-l_{5}\right)$ & 0 \\
\hline 6 & $-\left(a_{6}-l_{6}\right)$ & 0 & 0 \\
\hline
\end{tabular}

Matrices $\mathbf{A}_{i}$ and vectors $\mathbf{r}_{O i}=\left[x_{O i}, y_{O i}, z_{O i}\right]^{T}$ can be calculated by using transformation matrix $\mathbf{D}_{i}$ obtained in the last section. They are

$$
\begin{aligned}
& \mathbf{A}_{1}=\left[\begin{array}{ccc}
C_{1} & 0 & S_{1} \\
S_{1} & 0 & -C_{1} \\
0 & 1 & 0
\end{array}\right], \mathbf{r}_{O 1}=\left[\begin{array}{c}
0 \\
0 \\
d_{1}
\end{array}\right], \mathbf{A}_{2}=\left[\begin{array}{ccc}
C_{1} C_{2} & -C_{1} S_{2} & S_{1} \\
S_{1} C_{2} & -S_{1} S_{2} & -C_{1} \\
S_{2} & C_{2} & 0
\end{array}\right], \mathbf{r}_{O 2}=\left[\begin{array}{c}
a_{2} C_{1} C_{2} \\
a_{2} S_{1} C_{2} \\
a_{2} S_{2}+d_{1}
\end{array}\right] \\
& \mathbf{A}_{3}=\left[\begin{array}{ccc}
C_{1} C_{23} & -C_{1} S_{23} & S_{1} \\
S_{1} C_{23} & -S_{1} S_{23} & -C_{1} \\
S_{23} & C_{23} & 0
\end{array}\right], \mathbf{r}_{O 3}=\left[\begin{array}{c}
a_{2} C_{1} C_{2}+a_{3} C_{1} C_{23} \\
a_{2} S_{1} C_{2}+a_{3} S_{1} C_{23} \\
d_{1}+a_{2} S_{2}+a_{3} S_{23}
\end{array}\right] \\
& \mathbf{A}_{4}=\left[\begin{array}{ccc}
C_{1} C_{234} & -S_{1} & -C_{1} S_{234} \\
S_{1} C_{234} & C_{1} & -S_{1} S_{234} \\
S_{234} & 0 & C_{234}
\end{array}\right], \mathbf{r}_{O 4}=\left[\begin{array}{c}
a_{2} C_{1} C_{2}+a_{3} C_{1} C_{23} \\
a_{2} S_{1} C_{2}+a_{3} S_{1} C_{23} \\
d_{1}+a_{2} S_{2}+a_{3} S_{23}
\end{array}\right] \\
& \mathbf{A}_{5}=\left[\begin{array}{ccc}
C_{1} C_{234} C_{5}-S_{1} S_{5} & -C_{1} S_{234} & S_{1} C_{5}+C_{1} S_{5} C_{234} \\
S_{1} C_{234} C_{5}+C_{1} S_{5} & -S_{1} S_{234} & -C_{1} C_{5}+S_{1} S_{5} C_{234} \\
S_{234} C_{5} & C_{234} & S_{5} S_{234}
\end{array}\right] \\
& \mathbf{r}_{O 5}=\left[\begin{array}{c}
-d_{5} C_{1} S_{234}+a_{2} C_{1} C_{2}+a_{3} C_{1} C_{23} \\
-d_{5} S_{1} S_{234}+a_{2} S_{1} C_{2}+a_{3} S_{1} C_{23} \\
d_{1}+d_{5} C_{234}+a_{2} S_{2}+a_{3} S_{23}
\end{array}\right] \\
& \mathbf{A}_{6}=\left[\begin{array}{ccc}
-C_{6} S_{1} S_{5}-S_{6} C_{1} S_{234}+C_{6} C_{5} C_{1} C_{234} & S_{6} S_{1} S_{5}-C_{6} C_{1} S_{234}-S_{6} C_{5} C_{1} C_{234} & S_{1} C_{5}+S_{5} C_{1} C_{234} \\
C_{6} S_{5} C_{1}-S_{6} S_{1} S_{234}+C_{6} C_{5} S_{1} C_{234} & -S_{6} S_{5} C_{1}-C_{6} S_{1} S_{234}-S_{6} C_{5} S_{1} C_{234} & -C_{1} C_{5}+S_{1} S_{5} C_{234} \\
C_{5} C_{6} S_{234}+S_{6} C_{234} & -C_{5} S_{6} S_{234}+C_{6} C_{234} & S_{5} S_{234}
\end{array}\right] \\
& \mathbf{r}_{O 6}=\left[\begin{array}{c}
-a_{6} C_{6} S_{1} S_{5}+a_{2} C_{1} C_{2}+\left(-a_{6} S_{6} C_{1}-d_{5} C_{1}\right) S_{234}+a_{6} C_{6} C_{5} C_{1} C_{234}+a_{3} C_{1} C_{23} \\
a_{6} C_{6} S_{5} C_{1}+a_{2} S_{1} C_{2}+\left(-a_{6} S_{6} S_{1}-d_{5} S_{1}\right) S_{234}+a_{6} C_{6} C_{5} S_{1} C_{234}+a_{3} S_{1} C_{23} \\
a_{6} C_{5} C_{6} S_{234}+\left(a_{6} S_{6}+d_{5}\right) C_{234}+a_{3} S_{23}+a_{2} S_{2}+d_{1}
\end{array}\right]
\end{aligned}
$$

The angular velocities of the links with respect to the link-fixed coordinate frame can be calculated using the relationship [13]

$$
\tilde{\boldsymbol{\omega}}_{i}^{(i)}=\mathbf{A}_{i}^{T} \dot{\mathbf{A}}_{i}
$$


The use of Eq. (16) yields

$$
\begin{aligned}
& \boldsymbol{\omega}_{1}^{(1)}=\left[\begin{array}{c}
0 \\
\dot{q}_{1} \\
0
\end{array}\right], \boldsymbol{\omega}_{2}^{(2)}=\left[\begin{array}{c}
S_{2} \dot{q}_{1} \\
C_{2} \dot{q}_{1} \\
\dot{q}_{2}
\end{array}\right], \boldsymbol{\omega}_{3}^{(3)}=\left[\begin{array}{c}
S_{23} \dot{q}_{1} \\
C_{23} \dot{q}_{1} \\
\dot{q}_{2}+\dot{q}_{3}
\end{array}\right], \boldsymbol{\omega}_{4}^{(4)}=\left[\begin{array}{c}
S_{234} \dot{q}_{1} \\
-\left(\dot{q}_{2}-\dot{q}_{3}-\dot{q}_{4}\right) \\
C_{234} \dot{q}_{1}
\end{array}\right] \\
& \boldsymbol{\omega}_{5}^{(5)}=\left[\begin{array}{c}
C_{5} S_{234} \dot{q}_{1}-S_{5}\left(\dot{q}_{2}+\dot{q}_{3}+\dot{q}_{4}\right) \\
C_{234} \dot{q}_{1}+\dot{q}_{5} \\
S_{5} S_{234} \dot{q}_{1}+C_{5}\left(\dot{q}_{2}+\dot{q}_{3}+\dot{q}_{4}\right)
\end{array}\right] \\
& \boldsymbol{\omega}_{6}^{(6)}=\left[\begin{array}{c}
C_{5} C_{6} S_{234} \dot{q}_{1}+S_{6} C_{234} \dot{q}_{1}-S_{5} C_{6}\left(\dot{q}_{2}+\dot{q}_{3}+\dot{q}_{4}\right)+S_{6} \dot{q}_{5} \\
-C_{5} S_{6} S_{234} \dot{q}_{1}+C_{6} C_{234} \dot{q}_{1}+S_{5} S_{6}\left(\dot{q}_{2}+\dot{q}_{3}+\dot{q}_{4}\right)+C_{6} \dot{q}_{5} \\
\dot{q}_{6}+S_{5} S_{234} \dot{q}_{1}+C_{5}\left(\dot{q}_{2}+\dot{q}_{3}+\dot{q}_{4}\right)
\end{array}\right]
\end{aligned}
$$

Using the obtained expressions of vectors $\mathbf{r}_{i}$ and $\boldsymbol{\omega}_{i}^{(i)}$ we get Jacobian matrices

$$
\begin{aligned}
& \mathbf{J}_{T 1}=\frac{\partial \mathbf{r}_{1}}{\partial \mathbf{q}}=\left[\begin{array}{cccccc}
0 & 0 & 0 & 0 & 0 & 0 \\
0 & 0 & 0 & 0 & 0 & 0 \\
0 & 0 & 0 & 0 & 0 & 0
\end{array}\right] \\
& \mathbf{J}_{T 2}=\frac{\partial \mathbf{r}_{2}}{\partial \mathbf{q}}=\left[\begin{array}{cccccc}
-l_{2} S_{2} C_{2} & -l_{2} S_{2} C_{1} & 0 & 0 & 0 & 0 \\
l_{2} C_{1} C_{2} & -l_{2} S_{1} S_{2} & 0 & 0 & 0 & 0 \\
0 & l_{2} C_{2} & 0 & 0 & 0 & 0
\end{array}\right] \\
& \mathbf{J}_{T 3}=\frac{\partial \mathbf{r}_{3}}{\partial \mathbf{q}}=\left[\begin{array}{cccccc}
-l_{3} S_{1} C_{231}-a_{2} S_{1} C_{2} & -l_{3} C_{1} S_{23}-a_{2} C_{1} S_{2} & -l_{3} C_{1} S_{23} & 0 & 0 & 0 \\
l_{3} C_{1} C_{23}+a_{2} C_{1} C_{2} & -l_{3} S_{1} S_{23}-a_{2} S_{1} S_{2} & -l_{3} S_{1} S_{23} & 0 & 0 & 0 \\
0 & l_{3} C_{23}+a_{2} C_{2} & l_{3} C_{23} & 0 & 0 & 0
\end{array}\right] \\
& \mathbf{J}_{R 1}^{(1)}=\frac{\partial \boldsymbol{\omega}_{1}^{(1)}}{\partial \dot{\mathbf{q}}}=\left[\begin{array}{llllll}
0 & 0 & 0 & 0 & 0 & 0 \\
1 & 0 & 0 & 0 & 0 & 0 \\
0 & 0 & 0 & 0 & 0 & 0
\end{array}\right], \quad \mathbf{J}_{R 2}^{(2)}=\frac{\partial \boldsymbol{\omega}_{2}^{(2)}}{\partial \dot{\mathbf{q}}}=\left[\begin{array}{cccccc}
S_{2} & 0 & 0 & 0 & 0 & 0 \\
C_{2} & 0 & 0 & 0 & 0 & 0 \\
0 & 1 & 0 & 0 & 0 & 0
\end{array}\right] \text {, } \\
& \mathbf{J}_{R 3}^{(3)}=\frac{\partial \boldsymbol{\omega}_{3}^{(3)}}{\partial \dot{\mathbf{q}}}=\left[\begin{array}{cccccc}
S_{23} & 0 & 0 & 0 & 0 & 0 \\
C_{23} & 0 & 0 & 0 & 0 & 0 \\
0 & 1 & 1 & 0 & 0 & 0
\end{array}\right], \mathbf{J}_{R 4}^{(4)}=\frac{\partial \boldsymbol{\omega}_{4}^{(4)}}{\partial \dot{\mathbf{q}}}=\left[\begin{array}{cccccc}
S_{234} & 0 & 0 & 0 & 0 & 0 \\
0 & -1 & -1 & -1 & 0 & 0 \\
C_{234} & 0 & 0 & 0 & 0 & 0
\end{array}\right] \\
& \mathbf{J}_{R 5}^{(5)}=\frac{\partial \boldsymbol{\omega}_{5}^{(5)}}{\partial \dot{\mathbf{q}}}=\left[\begin{array}{cccccc}
S_{234} C_{5} & -S_{5} & -S_{5} & -S_{5} & 0 & 0 \\
C_{234} & 0 & 0 & 0 & 1 & 0 \\
S_{234} S_{5} & C_{5} & C_{5} & C_{5} & 0 & 0
\end{array}\right] \\
& \mathbf{J}_{R 6}^{(6)}=\frac{\partial \boldsymbol{\omega}_{6}^{(6)}}{\partial \dot{\mathbf{q}}}=\left[\begin{array}{cccccc}
S_{6} C_{234}+S_{234} C_{5} C_{6} & -S_{5} C_{6} & -S_{5} C_{6} & -S_{5} C_{6} & S_{6} & 0 \\
C_{6} C_{234}-S_{234} C_{5} S_{6} & S_{5} S_{6} & S_{5} S_{6} & S_{5} S_{6} & C_{6} & 0 \\
S_{234} S_{5} & C_{5} & C_{5} & C_{5} & 0 & 1
\end{array}\right] \text {. }
\end{aligned}
$$

Assumed that axes $x_{i}, \mathrm{y}_{i}, \mathrm{z}_{i}$ of the link-fixed coordinate frame are principal axes. The inertia matrix $\mathbf{I}_{i}^{(i)}$ of $i$-th link about the center of mass $C_{i}$, referred to the principal axes, can be written in the form

$$
\mathbf{I}_{i}^{(i)}=\left[\begin{array}{ccc}
I_{x i} & 0 & 0 \\
0 & I_{y i} & 0 \\
0 & 0 & I_{z i}
\end{array}\right]
$$


The potential energy of the system can be written in the form

$$
\boldsymbol{\Pi}=\sum_{i=1}^{6} m_{i} \overline{\mathbf{g}}^{T} \mathbf{r}_{i}
$$

where $\overline{\mathbf{g}}=[0,0,-g]^{T}$ with the gravity acceleration $g \approx 9.81 \mathrm{~m} / \mathrm{s}^{2}$.

$$
\begin{aligned}
\Pi= & m_{1} g l_{1}+m_{2} g\left(l_{2} S_{2}+d_{1}\right)+m_{3} g\left(l_{3} S_{23}+a_{2} S_{2}+d_{1}\right) \\
& +m_{4} g\left(l_{4} C_{234}+a_{2} S_{2}+a_{3} S_{23}+d_{1}\right) \\
& +m_{5} g\left(l_{5} C_{234}+a_{2} S_{2}+a_{3} S_{23}+d_{1}\right) \\
& +m_{6} g\left(l_{6} S_{6} C_{234}+l_{6} C_{5} C_{6} S_{234}+a_{2} S_{2}+a_{3} S_{23}+d_{5} C_{234}+d_{1}\right)
\end{aligned}
$$

Substituting Eqs. (17)-(24) into Eq. (12), we obtain the expression of the inertia matrix $\mathbf{M}(\mathbf{q})$ of the manipulator. Matrix $\mathbf{C}(\mathbf{q}, \dot{\mathbf{q}})$ can then be determined using Eq. (13). Substitution of Eq. (26) into (14) yields the gravity torque vector $\mathbf{g}(\mathbf{q})$. Finally, the joint torque vector $\boldsymbol{\tau}=\left[\tau_{1}, \tau_{2}, \tau_{3}, \tau_{4}, \tau_{5}, \tau_{6}\right]^{T}$ is given by Eq. (11). The formulation is implemented conveniently by means of the software packet MAPLE. However, the obtained expressions of $\mathbf{M}(\mathbf{q}), \mathbf{C}(\mathbf{q}, \dot{\mathbf{q}}), \mathbf{g}(\mathbf{q})$ and $\boldsymbol{\tau}$ can not be presented here in detail due to the complexity of formulae. The inertia parameters of the manipulator are given in Tab. 4 for the purpose of numerical calculation.

Table 4. Inertia parameters of the manipulator

\begin{tabular}{|l|l|l|l|l|l|}
\hline Link $i$ & $\begin{array}{l}m_{i} \\
(\mathrm{~kg})\end{array}$ & $\begin{array}{l}I_{x i} \\
\left(\mathrm{kgm}^{2}\right)\end{array}$ & $\begin{array}{l}I_{y i} \\
\left(\mathrm{kgm}^{2}\right)\end{array}$ & $\begin{array}{l}I_{z i} \\
\left(\mathrm{kgm}^{2}\right)\end{array}$ & $\begin{array}{l}l_{i} \\
(\mathrm{~m})\end{array}$ \\
\hline 1 & 2.0 & $4.0 \times 10^{-3}$ & $3.0 \times 10^{-3}$ & $1.0 \times 10^{-3}$ & 0.10 \\
\hline 2 & 0.9 & $0.2 \times 10^{-3}$ & $3.0 \times 10^{-3}$ & $3.0 \times 10^{-3}$ & 0.06 \\
\hline 3 & 1.2 & $0.5 \times 10^{-3}$ & $3.5 \times 10^{-3}$ & $4.0 \times 10^{-3}$ & 0.10 \\
\hline 4 & 1.1 & $0.6 \times 10^{-3}$ & $2.5 \times 10^{-3}$ & $3.5 \times 10^{-3}$ & 0.04 \\
\hline 5 & 0.5 & $0.7 \times 10^{-3}$ & $0.2 \times 10^{-3}$ & $0.3 \times 10^{-3}$ & 0.03 \\
\hline 6 & 0.05 & $0.3 \times 10^{-4}$ & $0.2 \times 10^{-4}$ & $0.1 \times 10^{-4}$ & 0.02 \\
\hline
\end{tabular}

\section{NUMERICAL EXAMPLE AND EXPERIMENTAL COMPARISON}

\subsection{Numerical example}

Now we consider a numerical example with a simple motion law of point $\mathrm{E}$ as shown in Fig. 3, which is described by the following time functions of coordinates

$$
x_{E}=0.2+0.12\left(1-\cos \frac{\pi}{4} t\right) \quad(m) ; \quad y_{E}=0 ; \quad z_{E}=0.14+0.12 \sin \frac{\pi}{4} t(m)
$$

The following initial values are chosen for the joint angles $\mathbf{q}: q_{1}(0)=0, q_{2}(0)=$ $1.0472, q_{3}(0)=3.5511, q_{4}(0)=2.1206, q_{5}(0)=2.0(\mathrm{rad})$. In addition, the motion of link 6 is assumed that $q_{6}=\pi / 2, \dot{q}_{6}=0$. Figs. $4-5$ show the calculating results of the inverse kinematics and dynamics corresponding to the given trajectory of point E in Eq. (27). 


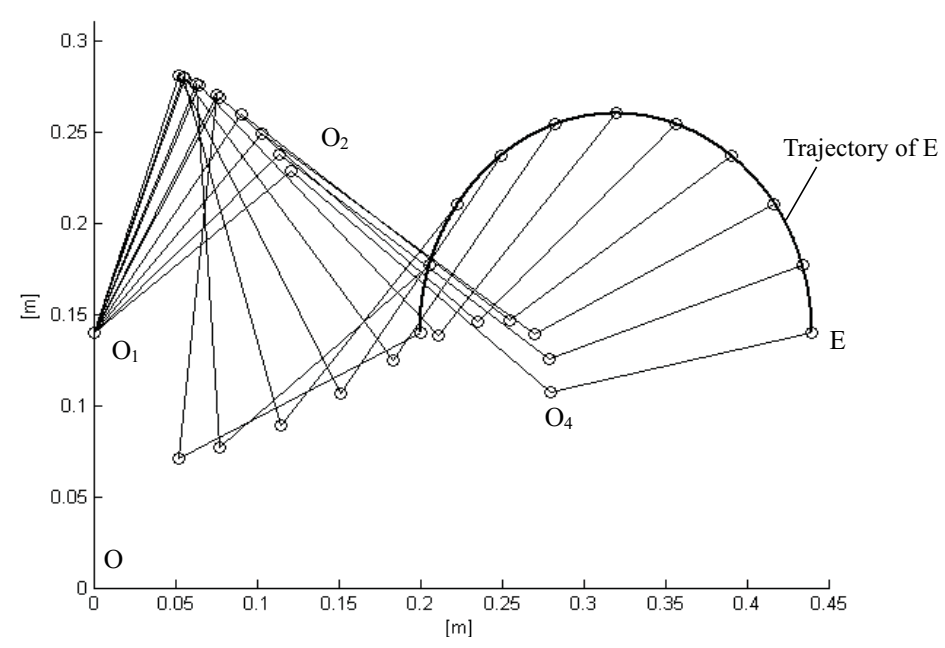

Fig. 3. Motion trajectory of point $\mathrm{E}$ and the position of the manipulator links

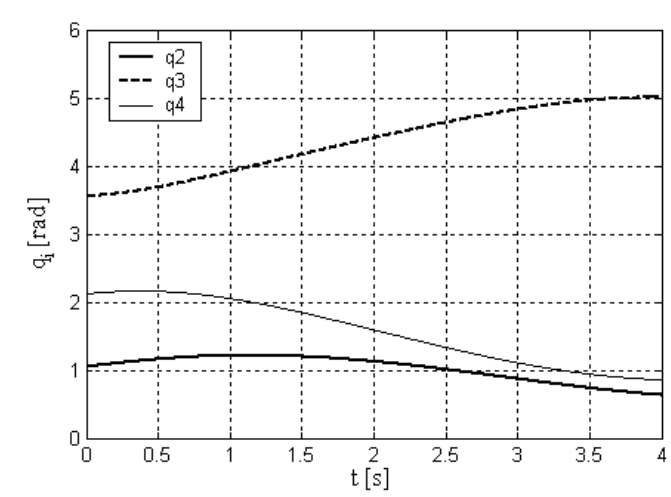

Fig. 4. Time curves of the joint angles

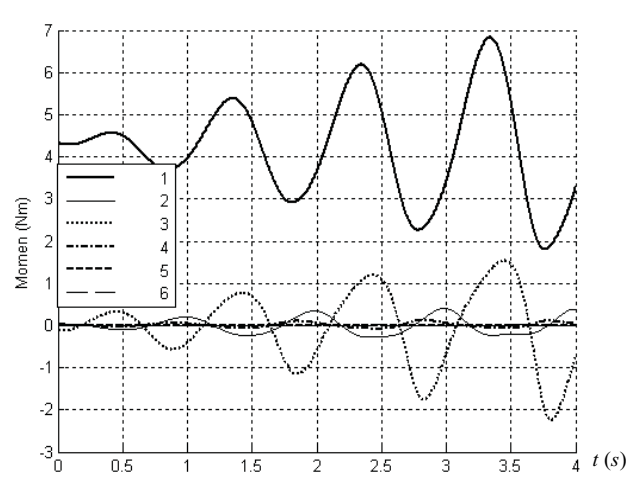

Fig. 5. Time curves of joint torques

\subsection{Experiment}

The experiment was done at the measuring manipulator designed and manufactured at Hanoi University of Technology. The major design parameter of the manipulator have been shown in Tabs. 1 and 4. During the test, the manipulator is controlled by a closed -loop control system to drive point E moving along the trajectory as shown in Fig. 3. The measurement of the real motion trajectory of point $\mathrm{E}$ was taken with optical transducers. The signal used in this study has been recorded for a duration of 4 seconds. Fig. 6 shows the experiment set-up. The measurement result is depicted in Fig. 7. As shown in Fig. 8, a good agreement is obtained between the calculation result and the experimental result.

\section{CONCLUSION}

This paper deals with the problem of inverse kinematics and dynamics of a measuring manipulator with kinematic redundancy which was designed and manufactured at 


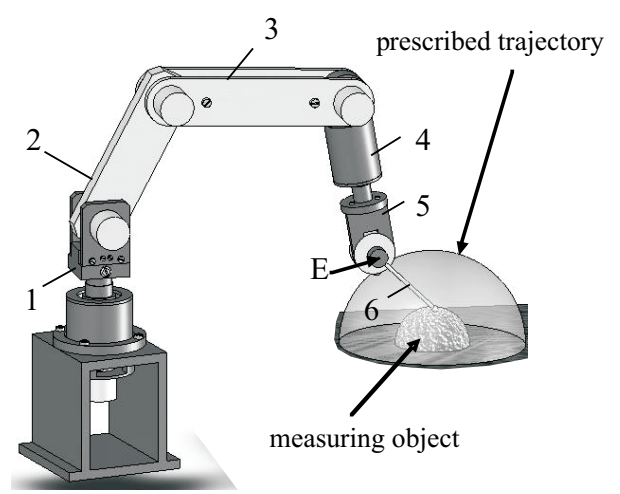

(a)

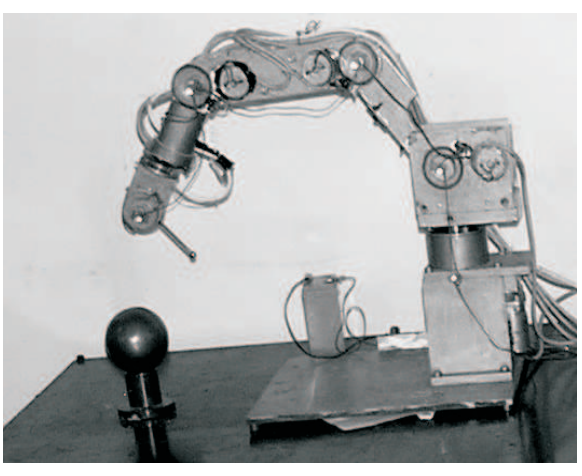

(b)

Fig. 6. (a) 3D-drawing, (b) the manufactured measuring manipulator

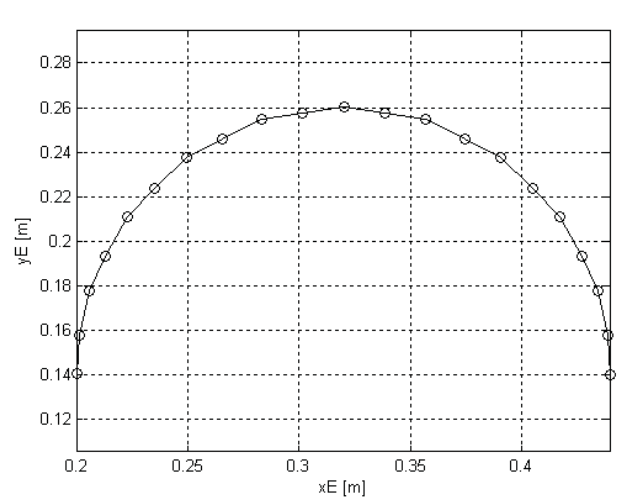

Fig. 7. The measured trajectory of point E

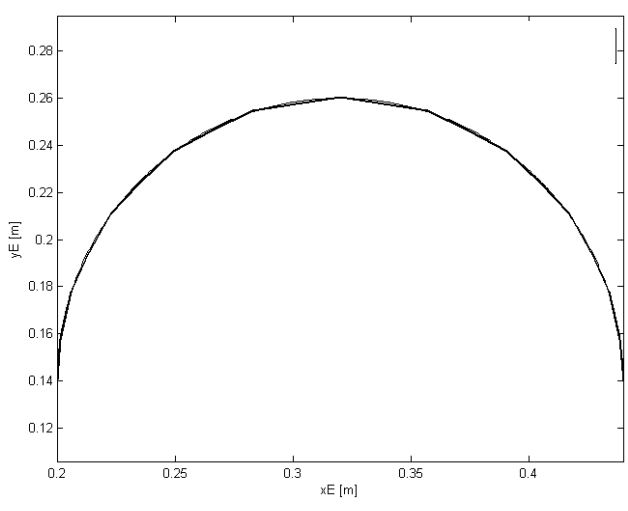

Fig. 8. Comparison between the calculation result (- - - - - ) and the experimental results $(-)$ for the trajectory of point $\mathrm{E}$

Hanoi University of Technology for measuring the geometric tolerance of surfaces of machining components. A comparison between the calculation result and the experimental measurement is also presented. It has been shown that the theory and algorithm used in this study provides a helpful tool to obtain exactly data for control tasks of redundant manipulators.

\section{ACKNOWLEDGMENT}

This paper was completed with the financial support given by the National Foundation for Science and Technology Development of Vietnam.

\section{REFERENCES}

[1] L. Sciavicco and B. Siciliano, A solution algorithm to the inverse kinematic problem of redundant manipulators. IEEE Journal of Robotics and Automation 4 (1988) 403-410. 
[2] P. Hsu, J. Hauser and S. Sastry, Dynamic Control of Redundant Manipulators, Journal of Robotic Systems 6 (1989) 133-148.

[3] I. D. Walker, The use of kinematic redundancy in reducing impact and contact effects in manipulation, Proc. IEEE International Conference on Robotics and Automation (1990), pp. 434-439.

[4] Y. Nakamura, Advanced Robotics: Redundancy and Optimization, Addison. Wesley, 1991.

[5] R. G. Roberts and A. A. Maciejewski, Repeatable Generalized Inverse Control Strategies for Kinematically Redundant Manipulators, IEEE Transactions on Automatic Control 38 (5) (1993) 689-699.

[6] T. Shamir and Y. Yomdin, Repeatability of Redundant Manipulators: Mathematical Solution of the Problem, IEEE Transactions on Automatic Control 33 (11) (1988) 1004-1009.

[7] M. W. Spong, M. Vidyasagar, Dynamics and Control of Robot Manipulators, John Wiley \& Sons, New York 1989.

[8] T. Yoshikawa, Foundation of Robotics Analysis and Control, MIT Press, Cambridge 1990.

[9] R. M. Murray, Z. Li and S. S. Sastry, A Mathematical Introduction to Robotic Manipulation, CRC Press, Boca Raton 2000.

[10] R. V. Patel and F. Shadpey, Control of Redundant Robot Manipulators, Theory and Experiments, Springer-Verlag, Berlin, Heidelberg 2005.

[11] Christian Ott, Cartesian Impedance Control of Redundant and Flexible-Joint Robots, Springer-Verlag, Berlin, Heidelberg 2008.

[12] Farbod Fahimi: Autonomous Robots, Modeling, Path Planning, and Control. Springer Science \& Business Media, LLC, New York 2009.

[13] Nguyen Van Khang, Multibody Dynamics (in Vietnamese), Science and Technique Publishing House, Hanoi 2007.

[14] Nguyen Van Khang, Do Tuan Anh, Nguyen Phong Dien, Tran Hoang Nam, Influence of trajectories on the joint torques of kinematically redundant manipulators, Vietnam Journal of Mechanics 29 (2) (2007) 65-72.

[15] Tran Hoang Nam, Inverse kinematic and dynamic analysis and control of redundant robots using a numerical algorithm correcting the increment of the vector of joint variables, $P h D$. thesis (manuscript in Vietnamese), Hanoi National University, 2009.

Received July 29, 2009

\section{PHÂN TÍCH ĐộNG HỌC VÀ ĐộNG LỰC HỌC NGƯợC CỦA RÔBỐT ĐO DƯ DẪN Đô̂NG BKHN-MCX-04}

Bài báo đề cập tới bài toán phân tích động học và động lực học ngược của tay máy rô bốt đo với đặc tính dư dẫn động. Rôbốt này được thiết kế và chế tạo tại Trường Đại học Bách khoa Hà nội để phục vụ cho các phép đo độ chính xác hình học của chi tiết gia công. Các kết quả tính toán đã được so sánh đối chiếu với các kết quả đo thực nghiệm. 